\title{
Global Passivity in Microscopic Thermodynamics
}

\author{
Raam Uzdin ${ }^{1,2}$ and Saar Rahav ${ }^{2}$ \\ ${ }^{1}$ Fritz, Haber Research Center for Molecular Dynamics, Hebrew University of Jerusalem, \\ Jerusalem 9190401, Israel \\ ${ }^{2}$ Schulich Faculty of Chemistry, Technion-Israel Institute of Technology, Haifa 3200008, Israel
}

(Received 18 February 2018; revised manuscript received 10 April 2018; published 12 June 2018; corrected 7 September 2018)

\begin{abstract}
The main thread that links classical thermodynamics and the thermodynamics of small quantum systems is the celebrated Clausius inequality form of the second law. However, its application to small quantum systems suffers from two cardinal problems. (i) The Clausius inequality does not hold when the system and environment are initially correlated - a commonly encountered scenario in microscopic setups. (ii) In some other cases, the Clausius inequality does not provide any useful information (e.g., in dephasing scenarios). We address these deficiencies by developing the notion of global passivity and employing it as a tool for deriving thermodynamic inequalities on observables. For initially uncorrelated thermal environments the global passivity framework recovers the Clausius inequality. More generally, global passivity provides an extension of the Clausius inequality that holds even in the presences of strong initial system-environment correlations. Crucially, the present framework provides additional thermodynamic bounds on expectation values. To illustrate the role of the additional bounds, we use them to detect unaccounted heat leaks and weak feedback operations ("Maxwell demons") that the Clausius inequality cannot detect. In addition, it is shown that global passivity can put practical upper and lower bounds on the buildup of system-environment correlations for dephasing interactions. Our findings are highly relevant for experiments in various systems such as ion traps, superconducting circuits, atoms in optical cavities, and more.
\end{abstract}

DOI: 10.1103/PhysRevX.8.021064

\section{INTRODUCTION}

Recent years have seen a surge of interest in the thermodynamics of small systems. Classical thermodynamics was developed for macroscopic systems that are weakly coupled to large environments. Technological advances now allow studying various processes in nanoscopic systems and it is of great interest to understand such processes from a thermodynamic point of view.

Applying thermodynamics to processes in small systems requires going beyond the assumptions and methodologies used in classical thermodynamics for several reasons. (1) The dynamics of microscopic systems is quantum, and questions regarding the thermodynamic role of quantum coherence, entanglement, and measurements become important. (2) The system-environment coupling cannot generally be assumed to be weak. As a result, the environment is modified by the system, and strong recurrences may

Published by the American Physical Society under the terms of the Creative Commons Attribution 4.0 International license. Further distribution of this work must maintain attribution to the author(s) and the published article's title, journal citation, and DOI.
Subject Areas: Quantum Physics,

Quantum Information,

Statistical Physics take place. (3) A non-negligible degree of initial systemenvironment correlation may be present, leading to effects such as heat flow from a cold subsystem to a hot one [1,2]. (4) Small quantum systems are easily taken out of equilibrium, and therefore their dynamics cannot be efficiently described by small number of quantities such as volume, average energy, and so on. In summary, it is of great interest to try to adapt thermodynamics to deal with some of these deviations from the assumptions used in classical macroscopic thermodynamics.

Several theoretical advances that extend thermodynamics were developed in the past few decades. Stochastic thermodynamics $[3,4]$ describes the fluctuations in thermodynamic characteristics of a process such as heat and work. These were found to satisfy the celebrated fluctuation theorems [5,6], a family of equalities that also hold far from equilibrium. Thermodynamic resource theory [7-10] studies the possible transformations a system can undergo by interacting with a thermal bath. Both theories have their limitations. Stochastic thermodynamics consider either systems decoupled from the environment, or systems coupled to macroscopic environments that remain in equilibrium during the process. Resource theory is limited to a specific set of operations called "thermal operations". 
The interest in the thermodynamics of small systems has led to several experimental realizations of microscopic processes that take after macroscopic thermodynamics. A heat engine with a single ion as a working fluid [11] as well as a three-ion absorption refrigerator [12] have been implemented. Algorithmic cooling [13] has been demonstrated in NMR [14]. In addition, quantum features of heat machines have also been recently observed in Nitrogen Vacancy centers in diamonds [15]. An experiment demonstrating the thermodynamic role of initial correlations was done in NMR [2]. While these experiments verify the validity of the second law for the smallest quantum systems, they also offer the possibility of testing new thermodynamic predictions such as the ones suggested in this paper.

In this work, we are mostly interested in processes where a small system of interest is coupled to other small systems that act as environments (Fig. 1). As a starting point, the small environments are assumed to be initially in Gibbs states $\rho_{0}^{(i)}=\exp \left(-\beta_{i} H_{i}\right) / \operatorname{tr}\left[\exp \left(-\beta_{i} H_{i}\right)\right]$, where $H_{i}$ is the Hamiltonian of environment $i$ and $\beta_{i}$ is its inverse temperatures. The system of interest can start in any state. Because of the microscopic size of the small environments, the interaction with the system will in general modify them, and they can substantially deviate from their initial Gibbs state. Moreover, they may develop a strong correlation to the system of interest. We call such small and initially thermal environments "microbaths" to distinguish them from macroscopic baths encountered in classical thermodynamics.

The dynamics of the whole setup containing the system of interest and all the microbaths is described by a global unitary dynamics (quantum evolution). More generally, the dynamics can be described by a statistical mixture of unitaries. Physically, a mixture of unitaries corresponds to the scenario where there is some noise in the controls generating the thermodynamic protocol. These global unitaries act on both the system and the microbaths (leading to heat flows), and can also do work. Our goal is to describe the thermodynamics of such processes even when the elements are initially correlated to each other.

One of the characteristics of thermodynamics is the appearance of inequalities. The second and third laws tell us that there are tasks that cannot be done. In the current setup the most relevant form of the second law is the Clausius inequality (CI). In the following, we show that the concept of passivity [16-20] can be extended and can be used to derive additional inequalities. These inequalities have several appealing features not exhibited by the CI: (1) they hold for systems that have some initial quantum or classical correlation to the environment, (2) they can set upper and lower bounds on the system-environment correlation buildup, and (3) they allow us to derive families of inequalities that can detect external tempering in the form of heat leaks or feedback (e.g., a Maxwell demon) even when the CI fails to detect the external intervention.

The flexibility and general applicability of the global passivity framework presented in this work come at a (a)

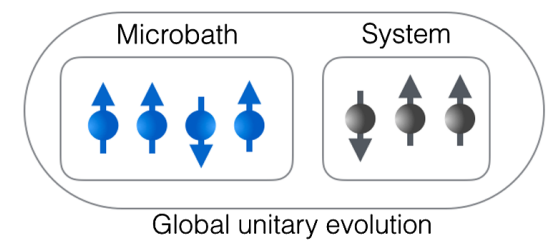

(b) Std. quant. thermo. setup

(c) General quant. thermo. setup
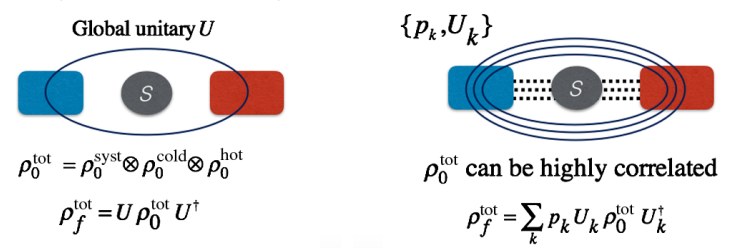

FIG. 1. (a) A typical scenario for our theory. A microbath with very small heat capacity (e.g., several spins) is initially prepared in a thermal state and then coupled to a system in a nonthermal state. In such scenarios the dynamics is highly non-Markovian and, in addition, the unitary transformation that generates the interactions may add or remove energy from the system-environment setup. (b) In the standard quantum thermodynamic setup all elements (microbaths and system) are initially uncorrelated and then a global unitary (a thermodynamic protocol) describes their interaction. (c) In this paper we consider any initial conditions including strong entanglement between the system and the microbaths. Moreover, we allow for a mixture of unitaries which include the possibilities of noise in the thermodynamic protocol.

price. Global passivity connects the initial state of the setup to the observable appearing in the resulting inequalities. As a result, in some cases the predictions obtained from these inequalities may involve nonintuitive observables. Nevertheless, the examples we give in this paper demonstrate that one can derive interesting new predictions in various important scenarios that were thus far outside the scope of the thermodynamic description. We believe that the flexibility of this framework is useful, and will lead to additional predictions on measurable quantities in nanoscopic setups.

In Sec. II, we introduce the notion of global passivity and use it to derive a version of the Clausius inequality that is valid in the presence of initial system-environment correlation. Several important examples are studied in detail. Section III uses the concept of global passivity to obtain a new type of inequalities. We exemplify the use of these new inequalities for detecting unaccounted heat leaks, for detecting the presence of "lazy" Maxwell demons, and for studying the buildup of system-environment correlation in a dephasing scenario. In these examples, the results obtained from global passivity are much more useful compared to the standard second law. We summarize our findings in Sec. IV.

\section{EXTENDING THE CLAUSIUS INEQUALITY USING GLOBAL PASSIVITY}

In this the section, we introduce the notion of global passivity and demonstrate how it can be used to derive various thermodynamic inequalities. Before doing so, we 
present the celebrated Clausius inequality formulation of the second law of thermodynamics. Under some restrictions, this formulation holds in some microscopic setups. We discuss its structure and its limitations when applied to microscopic systems.

\section{A. Clausius inequality in microscopic setups}

Historically, the second law was developed for macroscopic systems such as steam engines and large thermal reservoirs. However, it turns out that under some conditions one of its formulations, the Clausius inequality, also holds for small quantum systems interacting with each other and with external fields. Since this corresponds to the setup considered in this paper, the CI will serve as a natural reference for the new results described in this article.

Consider the setup shown in Fig. 1(b) in which a system of interest and several other small systems are prepared in an initially uncorrelated state of the form

$$
\rho_{0}^{\text {tot }}=\rho_{0}^{\text {sys }} \otimes e^{-\beta_{1} H_{1}} \otimes \cdots e^{-\beta_{N} H_{N}} / Z_{0},
$$

where $Z_{0}=\operatorname{tr}\left[e^{-\sum_{i=1}^{N} \beta_{i} H_{i}}\right]$ is a normalization factor, $H_{i}$ are the Hamiltonians of the systems that act as microbaths, where $\beta_{i}$ corresponds to the initial inverse temperatures. A thermodynamic process is realized via some timedependent global Hamiltonian that acts on all elements (not necessarily simultaneously). As a result, the setup evolves unitarily in time. The initial preparation distinguishes the system of interest, which can be in any initial state, from the microbaths that are initially in a thermal state. We note that this initial state is special due to the lack of correlation between the different elements.

The process described above satisfies a quantummicroscopic version of the CI [21-24],

$$
\Delta S^{\mathrm{sys}}+\sum_{k} \beta_{k} q_{k}^{\text {bath }} \geq 0,
$$

where $q_{k}=\Delta\left\langle H_{k}\right\rangle$ is the change in the average energy of microbath $k . \Delta S^{\text {sys }}=S\left(\rho_{f}^{\text {sys }}\right)-S\left(\rho_{0}^{\text {sys }}\right)$ is the change in the von Neumann entropy of the system $S^{\text {sys }}\left(\rho^{\text {sys }}\right) \doteq$ $-\operatorname{tr}\left[\rho^{\text {sys }} \ln \rho^{\text {sys }}\right]$, where $\rho^{\text {sys }}=\operatorname{tr}_{\text {baths }}\left[\rho^{\text {tot }}\right]$. This microscopic version goes beyond the assumptions of classical thermodynamics since the microbaths can be arbitrarily small in size and driven far from equilibrium during the process. However, it is restricted by the demand that all the elements are initially uncorrelated and that the microbaths start in thermal state.

One of the goals of this paper is to generalize Eq. (2) to the case where the elements are initially correlated. To make sure we find a plausible generalization we now take a closer look at the structure of the CI. The first term deals with changes in a quantity that is nonlinear in the density matrix $S(\rho)=-\operatorname{tr}[\rho \ln \rho]$. The nonlinearity appears in both the initial and the final state. As such, it is not an observable that can be directly measured, but rather a quantity that is calculated after $\rho$ has been evaluated via tomography of the system. Nevertheless, its informational interpretation makes it very useful. The second term, $\sum_{k} \beta_{k} q_{k}^{\text {bath }}$, is a measurable quantity that describes changes in expectation values.

With this combination of system information and observables, the CI neatly expresses the energyinformation relation that appears in fundamental processes such as Landauer's erasure, the Szilard engine, and reversible state preparation $[25,26]$. When extending the CI it is desirable to maintain this information-expectation value structure. See Ref. [26] for an extension of the second law that preserves the information-observable structure, and Refs. $[9,10]$ for an extension that does not.

Finally, we point out that using Eq. (1) and $\rho_{0}^{\text {env }} \doteq$ $\operatorname{tr}_{\text {sys }} \rho_{0}^{\text {tot }}$, the term $\sum_{k} \beta_{k} q_{k}^{\text {bath }}$ can be written as $\operatorname{tr}\left[\left(\rho_{f}^{\text {env }}-\rho_{0}^{\text {env }}\right)\left(-\ln \rho_{0}^{\text {env }}\right)\right]$. Using the notation

$$
\mathcal{B}^{\text {env }} \doteq-\ln \rho_{0}^{\text {env }},
$$

the standard CI Eq. (2) can be written in a form that will be useful later:

$$
\Delta S^{\text {sys }}+\Delta\left\langle\mathcal{B}^{\text {env }}\right\rangle \geq 0
$$

\section{B. Passivity and expectation values inequalities}

Passivity [16-20,27] is defined as follows: a timeindependent operator $\mathcal{A}$ and a density matrix $\rho$ are said to be passive with respect to each other if (i) $\rho$ and $\mathcal{A}$ are diagonal in the same basis (same eigenvectors) and (ii) in a basis sorted in increasing order of eigenvalues of $\mathcal{A}$, the eigenvalues of $\rho$ are decreasing. Since the eigenvalues of $\rho$ correspond to probabilities, it implies that when measuring $\mathcal{A}$ in a system prepared in a passive state, higher eigenvalues of $\mathcal{A}$ are less probable to be observed than lower eigenvalues. This is illustrated in Fig. 2(a).

Passive pairs $\left\{\mathcal{A}, \rho_{\mathcal{A}}\right\}$ satisfy an important inequality. Consider an initial passive state $\rho_{\mathcal{A}}$ with respect to $\mathcal{A}$ that is mapped to a final state via a unitary transformation,

$$
\rho_{f}=U \rho_{\mathcal{A}} U^{\dagger},
$$

or more generally by a mixture of unitaries,

$$
\rho_{f}=\sum_{k} p_{k} U_{k} \rho_{\mathcal{A}} U_{k}^{\dagger},
$$

where $p_{k}$ denotes the probability of executing the unitary $U_{k}$. The passivity of the initial state ensures that the expectation value $\langle\mathcal{A}\rangle_{f}=\operatorname{tr}\left(\rho_{f} \mathcal{A}\right)$ satisfies

$$
\langle\mathcal{A}\rangle_{f} \geq\langle\mathcal{A}\rangle_{\text {pass }}=\operatorname{tr}\left(\rho_{\mathcal{A}} \mathcal{A}\right) .
$$

Alternatively stated, the passive state gives the lowest expectation value achievable by mixture of unitary 
(a) Passive distribution w.r.t. operator $\boldsymbol{A}$

(b) nonpassive distribution

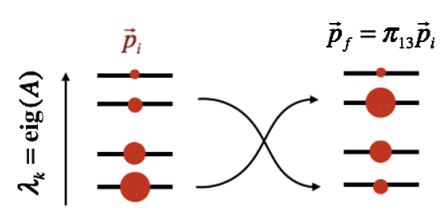

$\langle A\rangle_{i}=\sum_{k} p_{i, k} \lambda_{k}$ (c) Constructing more passive operators

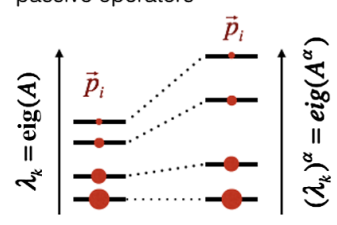

$A$ is passive w.r.t. $\vec{p}_{i}$ $\Rightarrow A^{\alpha}$ is passive w.r.t. $\vec{p}_{i}$

FIG. 2. (a) Passivity of a general Hermitian operator $\mathcal{A}$ with respect to (w.r.t.) initial density matrix $\rho_{0} . \vec{p}_{i}$ denote the eigenvalues of $\rho_{0}$. In passive distributions with respect to an observable $\mathcal{A}$, larger eigenvalues of $\mathcal{A}$ (measurement results $\lambda_{i}$ ) have lower probability to be observed (probability is given by the size of the circles). Panel (b) illustrates that starting from passive distribution, any permutation must increase the expectation value $\langle\mathcal{A}\rangle$. This implies that $\langle\mathcal{A}\rangle$ increases under a unitary operation $(\Delta\langle\mathcal{A}\rangle \geq 0)$. (c) If $\mathcal{A}$ is passive with respect to $\vec{p}_{i}$, then, $\mathcal{A}^{\alpha}$ is passive with respect to $\vec{p}_{i}$ for any $\alpha>0$.

transformations. The validity for mixture of unitaries follows from linearity and the validity for any single unitary Eq. (5). To see why Eq. (7) holds for any unitary, we use the fact that when a unitary operates on a diagonal matrix, then it holds that $\vec{p}_{f}$, the diagonal elements of the new density matrix, are related to the original diagonals $\vec{p}_{0}$ via a mixture of permutations $\Pi_{k}$ that are executed with some probability $\zeta_{k}: \vec{p}_{f}=\sum_{k} \zeta_{k} \Pi_{k} \vec{p}_{0}$. When the initial distribution is passive with respect to $\mathcal{A}, \vec{p}_{0}=\vec{p}_{\text {pass }}$, any permutation or a mixture of permutations (doubly stochastic map) will increase the expectation value of $\mathcal{A}$ [Fig. 2(b)]. Alternatively, one can use the proof presented in Ref. [18].

Traditionally in thermodynamics, the passivity inequality is related to the amount of work that can be extracted from a system using a transient unitary operation on the system. That is, the Hamiltonian of the system is driven by some external fields for some time, but in the end it returns to its original value (cyclic Hamiltonian). This maximum extractable work is called the ergotropy of the system [18], and it is obtained in a unitary process in which the system ends in a passive state with respect to the system Hamiltonian.

Among all the passive states with respect to the Hamiltonian, the Gibbs state has a special status. The Gibbs state is the only state that has the property of complete passivity with respect to the Hamiltonian [16]. A collection of $N$ copies of the system in a Gibbs state is also a passive state with respect to the total Hamiltonian. Thus, no unitary that acts on the $N$ copies (including unitaries that describe interactions between the copies) can be used to reduce the total energy. This provides a link to the second law, as it excludes the work extraction from a single thermal reservoir.

Motivated by work extraction, in previous studies of passivity [16-20,27] the operator $\mathcal{A}$ was the

Hamiltonian of the system (or of the environment [20]). The use of passivity in this paper is different from the standard one in three different ways. (1) Here, passivity is applied to the entire setup and not just to the system. We use the term "global passivity" to distinguish this scenario from the standard use of passivity that deals with work extraction from a specific element in the setup. In particular, the energy flows involved in global passivity include heat flows between the elements. (2) We apply passivity to various operators not just to Hamiltonians. (3) In traditional passivity the operator (the Hamiltonian) is given and the passive states are the focus of interest. In contrast, in this paper we ask the question, given an initial density matrix $\rho_{0}$, what are the operators $\mathcal{A}$ that are passive with respect to $\rho_{0}$ ? The motivation is that for such an operator $\mathcal{A}$ it is guaranteed that

$$
\Delta\langle\mathcal{A}\rangle=\operatorname{tr}\left[\left(\rho_{f}-\rho_{0}\right) \mathcal{A}\right] \geq 0
$$

for any $\rho_{f}$ generated from $\rho_{0}$ by a mixture of unitaries. These three differences lead to a new connection between passivity and the second law, and to new thermodynamic inequalities.

Before we apply Eq. (8) to thermodynamic scenarios, we describe two simple extensions of Eq. (8) that are used in this paper to obtain additional inequalities on expectation values of observables.

The first extension concerns operators which are not passive with respect to the initial state. Let $\rho_{f}$ be the density matrix obtained from $\rho_{0}$ by a mixture of unitaries. We are interested in the change of the expectation value of an operator $\tilde{\mathcal{A}}$. Even when the operator of interest $\tilde{\mathcal{A}}$ and the initial state $\rho_{0}$ are not passive with respect to each other we can write

$$
\begin{aligned}
\Delta\langle\tilde{\mathcal{A}}\rangle & \doteq\langle\tilde{\mathcal{A}}\rangle_{f}-\langle\tilde{\mathcal{A}}\rangle_{0} \\
& \geq\langle\tilde{\mathcal{A}}\rangle_{\min }-\langle\tilde{\mathcal{A}}\rangle_{0},
\end{aligned}
$$

where $\langle\tilde{\mathcal{A}}\rangle_{\min }$ is the minimal expectation value of $\tilde{\mathcal{A}}$ obtained by transforming $\rho_{0}$ via a mixture of unitaries. From the definition of passive states it holds that $\langle\tilde{\mathcal{A}}\rangle_{\min }=\operatorname{tr}\left[\rho_{\tilde{\mathcal{A}} \text { ass }} \tilde{\mathcal{A}}\right] \doteq\langle\tilde{\mathcal{A}}\rangle_{\tilde{\mathcal{A}} \text { pass }}$, where $\rho_{\tilde{\mathcal{A}} \text { pass }}$ is obtained from $\rho_{0}$ via a unitary rotation $V$ so that $\rho_{\tilde{\mathcal{A}} \text { pass }}$ is passive with respect to $\tilde{\mathcal{A}}$. Therefore, we obtain

$$
\Delta\langle\tilde{\mathcal{A}}\rangle \geq\langle\tilde{\mathcal{A}}\rangle_{\tilde{\mathcal{A}} \text { pass }}-\langle\tilde{\mathcal{A}}\rangle_{0}
$$

Importantly, the right-hand side is independent of $\rho_{f}$. Thus, it can be evaluated before running the experiment without knowing the details of the evolution. As shown in Sec. III C, Eq. (11) can be quite useful.

The second extension of passivity we exploit in this paper concerns the case where a passive operator with 
respect to the initial density matrix is given, and we want to find additional passive operators in order to set additional constraints on additional observable quantities. Let $\mathcal{A}$ be a passive operator with respect to $\rho_{0}$, and let $g$ be an analytic and monotonically increasing function in the interval between the smallest and largest eigenvalues of $\mathcal{A}$. It holds that the operator $g(\mathcal{A})$ is also passive with respect to $\rho_{0}$ and, therefore,

$$
\Delta\langle g(\mathcal{A})\rangle \geq 0
$$

for any mixture of unitaries. As we demonstrate later, these new inequalities are not just a restatement of Eq. (8) and they have different physical content. The proof of Eq. (12) immediately follows from the fact that $\mathcal{A}$ and $g(\mathcal{A})$ have the same eigenvectors and also the same eigenvalue ordering since monotonically increasing functions just "stretches" the spectrum [Fig. 2(c)] but does not change the order of the eigenvalues. As a result, any density matrix $\rho_{0}$ that is passive with respect to $\mathcal{A}$ is also automatically passive with respect to $g(\mathcal{A})$.

\section{Global passivity and its relation to Clausius inequality}

To introduce the notion of global passivity and its relation to the second law, we first examine a simplified scenario. In Sec. II D we treat the general case. Consider a setup that includes only initially uncorrelated microbaths. That is, there is no system that is initially in a nonthermal state. The initial state of the whole setup is therefore given by

$$
\rho_{0}^{\mathrm{tot}}=e^{-\beta_{1} H_{1}} \otimes \cdots e^{-\beta_{N} H_{N}} / Z_{0}
$$

where $Z_{0}=\operatorname{tr}\left[e^{-\beta_{1} H_{1}} \otimes \cdots e^{-\beta_{N} H_{N}}\right]$ is a normalization factor. This situation can describe an absorption refrigerator such as the one implemented in Ref. [12]. For this initial state, the $\mathrm{CI}$ reduces to

$$
\sum_{k} \beta_{k} q_{k}^{\text {bath }} \geq 0
$$

To apply passivity for this setup we look for operators that are passive with respect to $\rho_{0}^{\text {tot }}$. A simple and systematic way to achieve this is to use $\rho_{0}^{\text {tot }}$ for the construction of the passive operators. We start with the elementary choice

$$
\mathcal{B}^{\text {tot }} \doteq-\ln \rho_{0}^{\text {tot }}
$$

We emphasize that Eq. (15) defines a time-independent operator based on the initial state. In particular, the expectation value of this operator at time $t$ reads $\left\langle\mathcal{B}^{\text {tot }}\right\rangle=\operatorname{tr}\left[\rho^{\text {tot }}(t)\left(-\ln \rho_{0}^{\text {tot }}\right)\right]$. It is simple to verify that $\mathcal{B}^{\text {tot }}$ is $\rho_{0}^{\text {tot }}$ passive. By inverting Eq. (15) we get $\rho_{0}^{\text {tot }}=e^{-\mathcal{B}^{\text {tot }}}$, which immediately implies that larger eigenvalues of $\mathcal{B}^{\text {tot }}$ are associated with lower probabilities.

Next, the setup evolves by a global unitary or mixture of unitaries:

$$
\rho_{f}^{\mathrm{tot}}=\sum_{k} p_{k} U_{k} \rho_{0}^{\mathrm{tot}} U_{k}^{\dagger}
$$

From passivity of $\mathcal{B}^{\text {tot }}$ with respect to $\rho_{0}^{\text {tot }}$, we get the inequality

$$
\Delta\left\langle\mathcal{B}^{\mathrm{tot}}\right\rangle \geq 0
$$

For the specific initial state Eq. (13), $\mathcal{B}^{\text {tot }}=\beta_{k} H_{k}-\left(\ln Z_{0}\right) I$, where $I$ is the identity operator. As a result, Eq. (17) becomes $\sum \beta_{k} q_{k} \geq 0$, and we retrieve the result predicted from the CI for this setup.

As mentioned earlier, we call this approach global passivity since we apply passivity for the whole setup and not just to the system of interest. In standard passivity, the operator is fixed (the Hamiltonian of the system) and the quantity of interest is the passive state. Here, the state is fixed or given by the initial preparation of the setup, and we are interested in operators that are passive with respect to it. In that sense, the present approach is complementary to the one usually used in the literature on passivity [16-20,27].

The global passivity inequality Eq. (17) is valid also for a setup that includes a system with an arbitrary initial state Eq. (1). The resulting inequality is less tight than the CI. On the other hand, it involves quantities that are easier to evaluate since all quantities are linear in the final density matrix. Hence, there is no need to perform a full system tomography in order to evaluate $S^{\text {sys }}\left(\rho_{f}\right)$, which appears in the CI. Details can be found in Appendix A.

Next, to obtain the complete CI form Eq. (2) that includes the system entropy term, we introduce the passivity-divergence relation. Quite remarkably, we obtain that the passivity-divergence relation not only reproduces the full $\mathrm{CI}$ but also yields a version of the $\mathrm{CI}$ that is valid in the presence of initial system-environment correlation.

\section{Passivity-divergence relation and the initial correlation Clausius inequality}

In thermal machines and in various thermal processes (e.g., thermal state preparation [25]), there is often a system of interest that does not start in a thermal state. In such cases, the inequality Eq. (14) is not applicable and Eq. (2) has to be used. Next, we use a more powerful version of global passivity to obtain an inequality that distinguishes between a system and its environment. We emphasize that this is not just a rederivation of the CI since the inequality we obtain can handle initial correlation between the system and the environment (in contrast to the $\mathrm{CI}$ ). 
Let us consider a setup initially prepared in a state $\rho_{0}^{\text {tot }}$. In contrast to the assumption of lack of correlation between the system of the environment Eq. (1), we now allow for a general density matrix $\rho_{0}^{\text {tot }}$ that may contain classical and quantum correlations between the various elements of the setup [Fig. 1(c)]. The initial reduced state of the system is obtained by tracing out the environment $\rho_{0}^{\text {sys }}=\operatorname{tr}_{\text {env }} \rho_{0}^{\text {tot }}$. Similarly, the initial state of the environment is $\rho_{0}^{\text {env }}=\operatorname{tr}_{\text {sys }} \rho_{0}^{\text {tot }}$.

Our starting point is the following identity:

$$
\operatorname{tr}\left[\left(\rho_{2}-\rho_{1}\right)\left(-\ln \rho_{1}\right)\right] \equiv S\left(\rho_{2}\right)-S\left(\rho_{1}\right)+D\left(\rho_{2} \mid \rho_{1}\right),
$$

where

$$
D\left(\rho_{2} \mid \rho_{1}\right) \doteq \operatorname{tr}\left[\rho_{2}\left(\ln \rho_{2}-\ln \rho_{1}\right)\right] \geq 0
$$

is the quantum relative entropy. To connect it to the notion of global passivity, we set $\rho_{2}=\rho_{f}^{\text {tot }}, \rho_{1}=\rho_{0}^{\text {tot }} \equiv \exp \left(-\mathcal{B}^{\text {tot }}\right)$ in Eq. (18) and obtain

$$
\Delta\left\langle\mathcal{B}^{\mathrm{tot}}\right\rangle \equiv \Delta S^{\mathrm{tot}}+D\left(\rho_{f}^{\mathrm{tot}} \mid \rho_{0}^{\mathrm{tot}}\right) .
$$

We now focus on the first term in the right-hand side of Eq. (20) that describes the change of the total von Neumann entropy of the setup. If the evolution of the setup is given by an exact unitary transformation $\rho_{f}^{\text {tot }}=U \rho_{0}^{\text {tot }} U$, then $\Delta S^{\text {tot }}=$ $S\left(\rho_{f}^{\text {tot }}\right)-S\left(\rho_{0}^{\text {tot }}\right)=0$ [28]. When a mixture of unitaries Eq. (16) is applied to the setup, it holds that $\Delta S^{\text {tot }} \geq 0$. This follows from the fact that the von Neumann entropy is Schur concave [29], and $\rho_{0}^{\text {tot }}$ majorizes $\rho_{f}^{\text {tot }}$ [the majorization follows immediately from Eq. (16)]. Physically, a mixture of unitaries describes the dynamics in the presence of some noise or randomness in the protocol.

Using $\Delta S^{\text {tot }} \geq 0$ in Eq. (20), we obtain the passivitydivergence relation:

$$
\Delta\left\langle\mathcal{B}^{\mathrm{tot}}\right\rangle \geq D\left(\rho_{f}^{\mathrm{tot}} \mid \rho_{0}^{\mathrm{tot}}\right) .
$$

Since $D\left(\rho_{f}^{\text {tot }} \mid \rho_{0}^{\text {tot }}\right) \geq 0$, the passivity divergence relation Eq. (21) immediately implies the global passivity $\Delta\left\langle\mathcal{B}^{\text {tot }}\right\rangle \geq 0$. This is alternative proof of the global passivity inequality.

Relation Eq. (21) is expressed using global quantities that involve the whole setup. To derive an inequality that distinguishes between system and environment and has a clear connection to the $\mathrm{CI}$, we use the monotonicity property of the quantum relative entropy: the quantum relative entropy decreases when subsystems are traced out [30]. In particular, it holds that

$$
D\left(\rho_{f}^{\text {tot }} \mid \rho_{0}^{\text {tot }}\right) \geq D\left(\rho_{f}^{\text {sys }} \mid \rho_{0}^{\text {sys }}\right)
$$

for any $\rho_{f}^{\text {tot }}, \rho_{0}^{\text {tot }}$ (even if the system is correlated to the rest of the setup). As a result, we get

$$
\Delta\left\langle\mathcal{B}^{\text {tot }}\right\rangle \geq D\left(\rho_{f}^{\text {sys }} \mid \rho_{0}^{\text {sys }}\right) .
$$

Applying Eq. (18) to the system density matrix and using it in Eq. (23), we obtain the initial correlation Clausius inequality (ICCI),

$$
\Delta S^{\text {sys }}+\Delta\left[\left\langle\mathcal{B}^{\text {tot }}\right\rangle-\left\langle\mathcal{B}^{\text {sys }}\right\rangle\right] \geq 0
$$

where $\mathcal{B}^{\text {sys }}$ is defined as

$$
\mathcal{B}^{\text {sys }} \doteq-\ln \rho_{0}^{\text {sys }} .
$$

The ICCI immediately reduces to the CI when the system and the environment are initially uncorrelated, since in this case $\left\langle\mathcal{B}^{\text {tot }}\right\rangle-\left\langle\mathcal{B}^{\text {sys }}\right\rangle=\left\langle\mathcal{B}^{\text {env }}\right\rangle$. The ICCI Eq. (24) is one of the main results in this paper.

The ICCI can also be recast in a slightly different form,

$$
\Delta S^{\text {sys }}+\Delta\left\langle\mathcal{B}^{\text {env }}\right\rangle+\Delta\left\langle\mathcal{B}^{\text {corr }}\right\rangle \geq 0
$$

where

$$
\mathcal{B}^{\text {corr }} \doteq \mathcal{B}^{\text {tot }}-\mathcal{B}^{\text {sys }} \otimes I^{\text {env }}-I^{\text {sys }} \otimes \mathcal{B}^{\text {env }}
$$

where $I$ is the identity operator and $\mathcal{B}^{\text {corr }}$ is a Hermitian operator that identically vanishes when the system and environment are initially uncorrelated. As the other $\mathcal{B}$ operators, $\mathcal{B}^{\text {corr }}$ is a time-independent operator that is determined by $\rho_{0}^{\text {tot }}$.

We use the terminology correlation compatible Clausius inequality for two reasons. Firstly, it reduces to the CI when $\rho_{0}^{\text {tot }}=\rho_{0}^{\text {sys }} \otimes \rho_{0}^{\text {env }}$. Secondly, the ICCI has the same structure as that of the CI: it relates changes in the von Neumann entropy of the system of interest and changes in expectation values. The difference is that on top of the changes in the environment expectation values, there is also a change in expectation value related to the initial system-environment correlation.

There is a "price" for incorporating the initial systemenviroment correlation into the second law in the form of expectation values. For the same initial reduced state of the environment, the form of $\mathcal{B}^{\text {corr }}$ will depend on the initial correlations in $\rho_{0}^{\text {tot }}$. That is, for different correlations, different expectation values appear in the ICCI. To put this "price" in perspective, it is important to note that the relation between the initial state of the setup and the expectation values that appears in the ICCI is already present in the standard CI. The initial preparation of the environment in Gibbs states leads to the $\sum_{k} \beta_{k} q_{k}$ term in the CI. In the ICCI we get that not only the initial state of the environment determines the expectation values but also the correlation to the system.

The role of the new correlation operator can be experimentally studied in various setups such as ion traps, superconducting qubits, and more. One of the simplest 
(a)

$\rho_{0}^{\mathrm{tot}}=\left(\begin{array}{cccc}\ldots & & & \\ & \ldots & c & \\ & c^{*} & \ldots & \\ & & & \ldots\end{array}\right)$

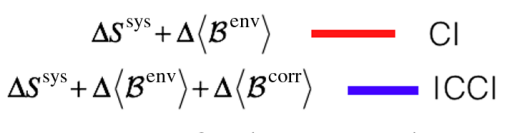

(b)
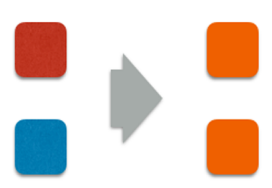

(c)

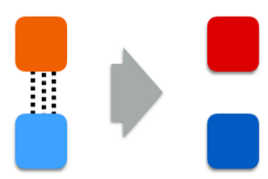

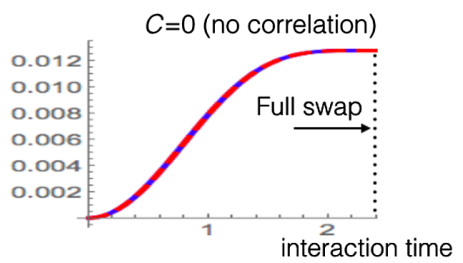

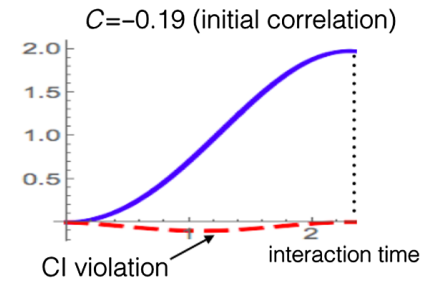

FIG. 3. The passivity-divergence relation offers an extension of the Clausius inequality (second law) that can handle initial correlation between environments or systems. (a) Hot and cold qubits are prepared with initial correlation in the form of coherence (superposition) between states $|01\rangle$ and $|10\rangle$ as shown by the form of the initial density. (b) In the absence of initial correlation $(C=0)$, an energy-conserving interaction makes the hot spin colder and the cold spin hotter. (c) In the presence of initial correlation $C=-0.19$, the same interaction makes the cold spin colder and the hot one hotter. All parameters are taken from a recent NMR experiment [2]. The top graph shows that the CI "entropy production" (red) and the left-hand side (lhs) of the ICCI (blue) have the same values for this case and both are positive at all time. However, in the presence of initial correlation (bottom graph), the lhs of Eq. (2) attains negative values, and the CI fails. In contrast, the ICCI remains valid (positive) at all times.

physical scenarios where this term strongly manifests is heat flow between two initially correlated spins. This has recently been experimentally demonstrated with NMR spins with quantum correlation [2]. The quantum correlation of the initial state [Fig. 3(a)] manifests in nonzero geometric quantum discord. The initially colder spin (c) will constitute the system, and the initially hotter $(h)$ will constitute the environment. Because of the initial correlation, heat can flow from the cold spin to the hot spin and make the cold spin colder and the hot spin hotter.

To test the ICCI we use the experimental parameters of Ref. [2] and plot [Fig. 3(b)] the CI (accumulated) entropy production: $\Delta S^{c}+\beta q_{h}$ and the left-hand side of the ICCI $\Delta S^{c}+\beta q_{h}+\Delta\left\langle\mathcal{B}^{\text {corr }}\right\rangle$. When there is no initial correlation both expressions are identical, as shown in Fig. 3(b). In the presence of correlation [Fig. 3(c)], the standard entropy production $\Delta S^{c}+\beta q_{h}$ becomes negative as the CI no longer holds for this scenario. In contrast, the ICCI expression $\Delta S^{c}+\beta q_{h}+\Delta\left\langle\mathcal{B}^{\text {corr }}\right\rangle$ remains positive at all times.

Finally, we emphasize that the essence of the ICCI is the inequality $D\left(\rho_{f}^{\text {tot }} \mid \rho_{0}^{\text {tot }}\right) \geq D\left(\rho_{f}^{\text {sys }} \mid \rho_{0}^{\text {sys }}\right)$ and not $\Delta S^{\text {tot }} \geq 0$.

$\Delta S^{\text {tot }}>0$ is simply another layer of randomness that is externally added by the noise in the controls. By using low noise control devices, an experimentalist can ensure that the evolution is carried out by a single unitary to very high accuracy. We also point out that the ICCI is a nonperturbative result and is valid for arbitrarily strong initial correlations and/or interactions between the elements.

In Sec. II E, we study an important thermodynamic scenario and work out explicitly the various terms in the ICCI. As a side comment, we point out that the derivation above for the case $\rho_{0}^{\text {tot }}=\rho_{0}^{\text {sys }} \otimes \rho_{0}^{\text {env }}$ constitutes an alternative to the previous derivations of the standard CI second law for microscopic quantum systems [21-24,31] (for the classical mechanics version, see Ref. [31]).

\section{E. ICCI for a coupled thermal state}

Next, we study an important case where the ICCI can be written more explicitly. Key quantities such as the interaction energy and the potential of mean force will emerge naturally in the derivation. Moreover, in Appendix B the structure obtained in this section is shown to be valid in much more general circumstances.

Let us consider a system that is permanently coupled to one of the small environments available in the setup by some interaction Hamiltonian $H_{I, 0}$. When preparing this small environment in a thermal state with inverse temperature $\beta_{h}$ (e.g., by weakly coupling it to a larger thermal bath that is later entirely decoupled from the setup), the system is also affected so the resulting system-environment state is $\rho_{0}^{h s}=\left(1 / Z_{h s}\right) e^{-\beta_{h}\left(H_{h}+H_{s}+H_{I, 0}\right)} . H_{s}$ and $H_{h}$ are the bare Hamiltonians of the system and the small environment, respectively. Note that the reduced state of the system or the environment is generally not Boltzmann distributed.

For generality, let us include another initially uncorrelated microbath at inverse temperature $\beta_{c}$. Thus, the initial density matrix is

$$
\rho_{0}^{\mathrm{tot}}=\frac{1}{Z_{h s} Z_{c}} e^{-\beta_{h}\left(H_{h}+H_{s}+H_{I, 0}\right)} e^{-\beta_{c} H_{c}}
$$

For this setup the ICCI Eq. (24) yields the following inequality:

$$
\begin{aligned}
& \Delta S^{\mathrm{sys}}+\beta_{c} q_{c}+\beta_{h} q_{h}+\beta_{h}\left[\Delta\left\langle H_{I, 0}\right\rangle+\Delta\left\langle H_{s}-H_{s}^{\mathrm{eff}}\right\rangle\right] \\
& \quad \geq 0
\end{aligned}
$$

As before, $q_{c}, q_{h}$ are the change in the average "bare" energies of the microbaths $\left\langle H_{c}\right\rangle,\left\langle H_{h}\right\rangle$ and

$$
H_{s}^{\mathrm{eff}} \doteq-\frac{1}{\beta_{h}} \ln \rho_{0}^{\mathrm{sys}}=-\frac{1}{\beta_{h}} \ln \operatorname{tr}_{h} e^{-\beta_{h}\left(H_{s}+H_{I, 0}+H_{h}\right)}
$$


The quantity $H_{s}^{\text {eff }}-H_{s}$ is known as the potential of means force [32] or as the solvation potential [33]. The first three terms in Eq. (28) are the "bare" terms that appear in the Clausius inequality. The fourth term, $\Delta\left\langle H_{I, 0}\right\rangle$, originates from the ICCI and it expresses the change in the interaction energy during the process. The last term in Eq. (28), $\Delta\left(H_{s}-H_{s}^{\text {eff }}\right)$, is the change in the "dressing energy" of the system. Because of the non-negligible interaction with the environment, the system is not initially in the thermal state of the bare system Hamiltonian.

Recently, it was brought to our attention that the ICCI for the coupled thermal state has some similarity to a recent classical formulation of the second law [33] for a very similar scenario. Despite the similarity there are quite a few differences between our result, Eq. (28), and Ref. [33]. We point out the two most important ones. First, our result is valid also in the presence of coherences (in the energy basis) and quantum correlations that arise from the initial system-environment coupling. Second, CI-like inequalities that are derived from fluctuation theorems [33-35] involve the equilibrium entropy and equilibrium free energy. In contrast, our result involves the von Neumann entropy of the nonequilibrium state and its corresponding nonequilibrium free energy $[24,36]$.

In Refs. [37,38], appealing treatments of initial correlations were presented in terms of changes in mutual information and conditional entropy. Such quantities cannot be expressed as expectation values (observables), and their evaluation requires costly, or even impractical, systemenvironment tomography. In contrast, in the ICCI, the initial correlation manifests only in terms of measurable expectation values. Furthermore, Ref. [37] assumes large environment, the availability of an external ancilla, and the feasibility of global operations on many copies of the setup. None of these assumptions are needed for the ICCI. Finally, Ref. [37] requires locally thermal states and therefore cannot handle the important initial states such as the coupled thermal state studied above Eq. (27). In Ref. [39], an interesting exchange fluctuation theorem is suggested. However, this approach is valid only for systems that are initially in local equilibrium. Moreover, it has no entropy term as in the CI and the ICCI. Thus, while Refs. [37-39] constitute important contributions to the field, the findings in these studies do not overlap with the results presented in this section. In particular, in Sec. III we derive additional inequalities that were not obtained in Refs. [37-39].

Equation (28) was derived for a setup prepared in a coupled thermal state Eq. (27). Its form seems to be rather different from the more general ICCI Eq. (24). However, in Appendix B we show that the same form also exists for a general initial state. In Appendix $C$ we study in more detail two physically interesting system-environment interactions. The first is an energy-conserving creation annihilation swaplike interaction, and the second is a dephasing interaction.

\section{ADDITIONAL PASSIVITY INEQUALITIES AND THEIR APPLICATION}

\section{A. Using passivity to generate new thermodynamic inequalities}

In Sec. II B it was shown that if an operator $\mathcal{A}$ is passive with respect to $\rho_{0}$, then $g(\mathcal{A})$ is also a passive operator with respect to $\rho_{0}$, where $g(x)$ is a monotonically increasing function in the domain covering the eigenvalues of $\mathcal{A}$. As shown before, the operator $\mathcal{B}_{\text {tot }}=-\ln \rho_{0}$ is passive with respect to $\rho_{0}^{\text {tot }}$ for any thermodynamic protocol. Its eigenvalues are non-negative. The function $g(x)=x^{\alpha}$ is an increasing function for $x \geq 0$ and $\alpha>0$. As a result, we get a new family of global passivity inequalities,

$$
\Delta\left\langle\mathcal{B}^{\alpha}\right\rangle \geq 0
$$

for any $\alpha>0$ and for any thermodynamic protocol Eq. (6). For brevity, in Sec. III "tot" is omitted and $\mathcal{B}$ will always refer to the whole setup. The $\alpha=1$ case was shown earlier to coincide with the CI in the case of initially uncoupled microbaths that interact with each other. Below, we demonstrate using three examples that the inequalities Eq. (30) have different physical content for different values of $\alpha$. This means that inequalities with $\alpha \neq 1$ carry information that is absent in the CI. We also note that different $\alpha$ inequalities obey a hierarchical structure, as shown in Appendix D.

\section{B. Heat leaks detection}

Let us assume that we are given a quantum chip (e.g., superconducting circuit with several qubits). The unitary operation that the chip is supposed to carry out is unknown to us, but we do have information on the number of qubits, qudits, and so on that constitute the input and output of the device. The thermodynamic challenge we face is to verify if this chip is isolated from the surroundings (for all practical purpose), or perhaps it interacts with some unaccounted for (hidden) heat bath, e.g., via spontaneous emission or thermalization to the substrate temperature. Note that the unitary implemented by the chip is in general not energy conserving (involves work). Hence, energy conservation cannot be used to detect the heat leak to the hidden environment.

One possible approach is to perform state tomography of the final state of the setup and check if the eigenvalues of the density matrix have been modified by an external agent. However, tomography of several qubits is very hard to perform and impractical for dozens of qubits. This method of detecting heat leaks is therefore very costly.

Another possibility is to use the second law. We prepare the input state in a product of thermal states $\rho_{0}^{\text {tot }}=$ $e^{-\sum \beta_{i} H_{i}} / Z$. According to the CI Eq. (2) the final density matrix satisfies $\sum_{i} \beta_{i} \operatorname{tr}\left[\rho_{f}^{\text {tot }} H_{i}\right] \geq \sum_{i} \beta_{i} \operatorname{tr}\left[\rho_{0}^{\text {tot }} H_{i}\right]$. Indeed, if this inequality does not hold, one can deduce that there is a 


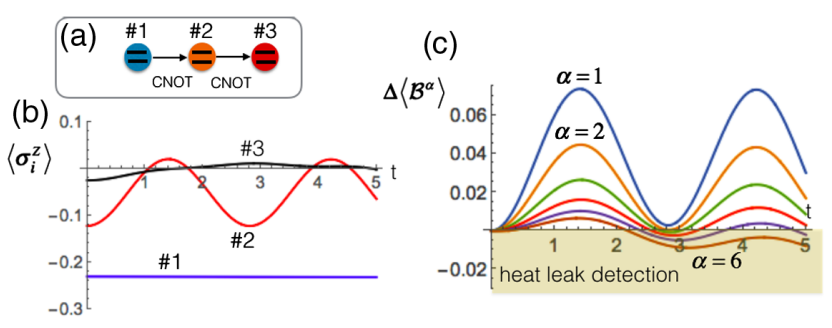

FIG. 4. (a) Heat leak detection in a three-qubit circuit with two CNOT interactions. The qubits also undergo decay to the ground state at a rate $\gamma=1 / 1000$. This weak decay is not visible in the polarization dynamics shown in (b). For example, the polarization of qubit 1 (blue) looks perfectly flat. Panel (c) shows a validity check of the inequalities Eq. (30). Since the $\alpha=1$ curve that corresponds to the CI is always positive for the depicted time, it cannot be used to detect the heat leak. On the other hand, the $\alpha=5$ and $\alpha=6$ global passivity inequalities are violated in less than $3 / 1000$ of the decay time (which is $1 / \gamma=1000$ ).

heat leak in progress. Crucially, if the $\mathrm{CI}$ is not violated, no conclusion can be made, as it possible that the heat leak is too small to be detected by the CI.

The global passivity inequalities Eq. (30) suggest a third possibility. A heat leak from the setup (chip) to some unaccounted environment cannot be described as a mixture of unitary on the setup only. Thus, in the presence of a heat leak from the chip, inequalities Eq. (30) may be violated and indicate the presence of a heat leak.

As an example, we study a three-qubit circuit with a CNOT interaction Hamiltonian between qubits 1 and 2, and qubits 2 and 3 [Fig. 4(a)]. The CNOT interaction strength is $\epsilon=1$, so the CNOT operation is implemented in a time $\hbar / \epsilon$. The two CNOT interactions operate simultaneously. In addition, there is a heat leak to a zero temperature bath (e.g., spontaneous emission) at a rate $\gamma=\epsilon / 1000$. That is, 1000 CNOT cycles pass by before the decay becomes dominant. Nevertheless, our goal is to detect the heat leak as soon as possible since the chip typically operates only for a very short time with respect to $1 / \gamma$.

The initial density matrix is $\rho_{0}^{\text {tot }}=e^{-\sum \beta_{i} \sigma_{z}^{(i)}} / Z$, with $\beta_{1}=1, \beta_{2}=0.5, \beta_{3}=0.1$. Figure 4 (b) shows that for the depicted time the effect of the decay on the polarization of the qubits is not visible. In Fig. 4(c) we plot the values of $\Delta\left\langle B^{\alpha}\right\rangle$ as a function of time for various $\alpha$ values. When the curves take negative values the heat leak is detected. The plot shows that larger $\alpha$ values can detect the heat leak much sooner compared to the $\alpha=1$ (which in this case equivalent to the $\mathrm{CI}$ ). It is important to emphasize that to evaluate $\Delta\left\langle\mathcal{B}^{\alpha}\right\rangle$ only local energy measurements $\left\{E_{1}, E_{2}, E_{3}\right\}$ are needed in each realization (in each run of the experiment). $\left\langle\mathcal{B}^{\alpha}\right\rangle$ is obtained by the mean value of $\left(\beta_{1} E_{1}+\beta_{2} E_{2}+\beta_{3} E_{3}\right)^{\alpha}$ in many runs. This is a much less demanding task compared to a three-qubit tomography that involves many nonlocal measurements.

These results show that different values of $\alpha$ contain different physical information. Moreover, it raises the interesting question of the existence of sufficient conditions for guaranteed detection of any heat leak.

\section{Bounds on system-environment correlation in a dephasing process}

As an additional example for the usefulness of the additional inequalities Eq. (30), let us consider the case where a system is coupled to the environment via a dephasing interaction. A dephasing interaction $H_{I}$ satisfies $\left[H_{I}, H_{\text {sys }}\right]=0$, and as a result it cannot affect the energy populations of the system. We also assume that the interaction does not change the energy populations of the environment $\left[H_{I}, H_{\text {env }}\right]=0$. In summary, the reduced energy distributions of both the system and environment are conserved (i.e., all energy moments are conserved).

In our example, the system is a single spin with a Hamiltonian $H_{s}=\sigma_{z}$. This spin is prepared with some initial coherence in the $x$ direction so that $\rho_{0}^{\text {sys }}=$ $e^{-\beta_{x} \sigma_{x}} / Z_{\text {sys }}$ where $\beta_{x}$ is dimensionless. As an environment, we take a three-spin microbath at inverse temperature $\beta$ [see inset in Fig. 5(a)] and a Hamiltonian $H_{\text {env }}=\sum_{i=1}^{3} \sigma_{z}^{(i)}$. The system and the microbath are initially uncorrelated. The total initial density matrix is

$$
\rho_{0}^{\mathrm{tot}}=e^{-\beta H_{\mathrm{env}} \otimes I_{s}-\beta_{x} I_{\mathrm{env}} \otimes \sigma_{x}} / Z,
$$
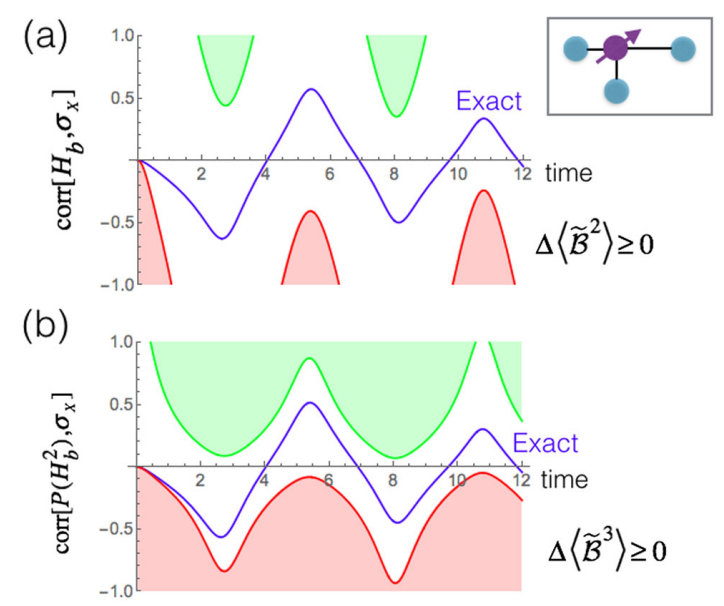

FIG. 5. Bounds on the system-environment correlation dynamics derived from the global passivity inequalities Eq. (30). (a) The blue curve depicts corr $\left[H_{\mathrm{env}}, \sigma_{x}\right]$ between the energy of a threespin microbath and the coherence $\left\langle\sigma_{x}\right\rangle$ of a one-spin system (see upper right-hand box) as a function of time under a dephasingtype interaction. The red and green shaded area show lower and upper bound on this correlation as obtained from the $\alpha=2$ global passivity inequality. (b) The $\alpha=3$ passivity inequality leads to even tighter bound (red) on the correlation to higher energy moments of the environment $P\left(H_{\text {env }}^{2}\right)=\beta_{x}^{2} \beta \tilde{H}_{\mathrm{env}}+\left(\beta H_{\mathrm{env}}\right)^{2}$, where $\beta$ is the temperature of the environment and $\beta_{x}$ determines the magnitude of the initial coherence of the system. 
where $I_{\mathrm{env}}, I_{s}$ are identity operators in the Hilbert space of the microbath and the system, and $Z$ normalizes the density matrix.

For convenience we define a shifted version of $\hat{B}$,

$$
\begin{gathered}
\tilde{\mathcal{B}}=\beta \tilde{H}_{\mathrm{env}}+\beta_{x} \tilde{\sigma}_{x}, \\
\tilde{H}_{\mathrm{env}}=H_{\mathrm{env}} \otimes I_{s}-E_{\mathrm{env}, 0} I, \\
\tilde{\sigma}_{x}=I_{\mathrm{env}} \otimes \sigma_{x}+1 / 2 I,
\end{gathered}
$$

where $I=I_{\mathrm{env}} \otimes I_{s}$ is the identity operator in the joint system-microbath space, and $E_{\text {env, } 0}$ is the ground state energy of the environment. Now, $\tilde{H}_{\text {env }}, \tilde{\sigma}_{\text {env }}$, and $\tilde{\mathcal{B}}$ are all positive operators, and in addition it holds that $\tilde{\sigma}_{x}^{n}=\tilde{\sigma_{x}}$, which will be useful later on. This shift is needed to ensure that powers of $\tilde{\mathcal{B}}$ will remain passive with respect to $\rho_{0}^{\text {tot }}$. For example, if the operator is shifted so that it contains both positive and negative eigenvalues, then squaring it will change the eigenvalue's order and destroy passivity with respect to $\rho_{0}^{\text {tot }}$. Alternatively stated, $g=x^{2}$ is increasing only for $x>0$, so $\tilde{\mathcal{B}}$ must be non-negative for even orders of Eq. (30) to be passive.

Next, we want to find a bound on the covariance between the energy of the microbath and the $\sigma_{x}$ values of the system at time $t$, i.e., $\operatorname{cov}\left[H_{\text {env }}, \sigma_{x}\right]_{t}=\left\langle H_{\text {env }} \sigma_{x}\right\rangle_{t}-\left\langle H_{\text {env }}\right\rangle\left\langle\sigma_{x}\right\rangle_{t}$. Since $\left\langle H_{\text {env }}\right\rangle=$ const and $\left\langle\sigma_{x}\right\rangle$ is easy to measure, the nontrivial term is $\left\langle H_{\mathrm{env}} \sigma_{x}\right\rangle_{t}$. This term can be bounded using Eq. (30). After some algebra, the $\alpha=2$ inequality $\Delta\left\langle\left(\beta \tilde{H}_{\mathrm{env}}+\beta_{s}{\tilde{\sigma_{x}}}^{2}\right\rangle \geq 0\right.$ leads to

$$
2 \beta \beta_{x} \Delta\left\langle\tilde{H}_{\mathrm{env}} \tilde{\sigma}_{x}\right\rangle \geq \beta_{x}^{2} \Delta\left\langle\tilde{\sigma}_{x}^{2}\right\rangle=\beta_{x}^{2} \Delta\left\langle\tilde{\sigma}_{x}\right\rangle .
$$

Subtracting $\left\langle H_{\text {env }}\right\rangle\left\langle\sigma_{x}\right\rangle_{t}-\left\langle H_{\text {env }}\right\rangle\left\langle\sigma_{x}\right\rangle_{0}$, we get

$$
\operatorname{cov}\left[H_{\mathrm{env}}, \sigma_{x}\right]_{t} \geq\left(\frac{\beta_{x}}{\beta}-\left\langle H_{\mathrm{env}}\right\rangle\right) \Delta\left\langle\tilde{\sigma}_{x}\right\rangle .
$$

We now turn to consider Eq. (30) with $\alpha=3$. Some straightforward algebraic manipulations result in

$$
\operatorname{cov}\left[P\left(\tilde{H}_{\text {env }}^{2}\right), \tilde{\sigma}_{x}\right]_{t} \geq c_{0} \Delta\left\langle\tilde{\sigma}_{x}\right\rangle,
$$

where

$$
P\left(\tilde{H}_{\mathrm{env}}^{2}\right)=\left(\beta \tilde{H}_{\mathrm{env}}\right)^{2}+\beta_{x}^{2}\left(\beta \tilde{H}_{\mathrm{env}}\right),
$$

and $c_{0}=\left[\frac{1}{3} \beta_{x}^{2}-\left\langle\beta^{2} \tilde{H}_{\mathrm{env}}^{2}+\beta_{x}^{2} \beta \tilde{H}_{\mathrm{env}}\right\rangle\right]$. Because of the dephasing interaction, $c_{0}$ is fixed in time and is process independent.

Equations (36) and (37) bound the buildup of system-environment covariance. To test these thermodynamic bounds numerically we choose the interaction Hamiltonian $H_{I}=\sum_{i=1}^{3} \xi_{i} \sigma_{z}^{(i)} \otimes \sigma_{z}^{\text {syst }}$, where $\xi_{1}=0.7$, $\xi_{2}=0.5, \xi_{3}=0.3$, that mimics a coupling that depends on the proximity of each microbath spin to the system. The blue curve in Fig. 5(a) shows the exact evolution of the correlation function corr $\left[H_{\text {env }}, \sigma_{x}\right]=\operatorname{cov}\left[H_{\text {env }}, \sigma_{x}\right]_{t} /$ $\sqrt{\operatorname{Var}\left(H_{\text {env }}\right) \operatorname{Var}\left(\sigma_{x}\right)}$. The red shaded areas show the lower bound Eq. (36) obtained from the $\alpha=2$ global passivity inequality. Figure 5(b) plots the correlation function $\operatorname{corr}\left[P\left(\tilde{H}_{\text {env }}^{2}\right), \sigma_{x}\right]=\operatorname{cov}\left[P\left(\tilde{H}_{\text {env }}^{2}\right), \sigma_{x}\right]_{t} /$ $\sqrt{\operatorname{Var}\left[P\left(\tilde{H}_{\text {env }}^{2}\right)\right] \operatorname{Var}\left(\sigma_{x}\right)}$ (blue curve) and the lower bound Eq. (37) obtained from the $\alpha=3$ global passivity inequality.

Using the $\alpha$ global passivity inequalitiess Eq. (30) and (11), it is also possible to get other types of bounds. In the example above, by applying a $\pi$ pulse (a unitary transformation) we obtain $\rho_{1}$, which is identical to $\rho_{0}$, but the system spin points in the opposite direction $\sigma_{x} \rightarrow-\sigma_{x}$. As a result, we get an upper bound on the systemenvironment covariance as shown by the green shaded areas in Fig. 5. We note that for small setups, upper bounds can also be obtained from the hierarchy relation described in Appendix D.

\section{Detecting evasive feedback demons}

A feedback is an operation applied to the system of interest, where the type of operation to be applied depends on the present state of the system. That is, the system is first measured and then different operations are executed depending on the measurement result. It is well known that feedback can completely change the thermodynamics of a process, and has to be properly accounted for. The most well-known example is Maxwell's celebrated demon [40]. In this thought experiment feedback is used to separate cold molecules from hot molecules, and thereby "violate" the second law of thermodynamics. A more modern viewpoint suggests that the second law holds if the information gained by the measurement is taken into account, or if the demon and the system are treated jointly as one big setup. The thermodynamics of information has become an active research field with many theoretical advances [41]. Several experimental realizations have also been reported [42-45].

In this section, we study the operation of a "lazy Maxwell demon." As in Maxwell's original thought experiment, the demon attempts to create an "anomalous heat flow" where heat flows from the cold environment the hot environment. Since the second law forbids such a heat flow in the absence of a feedback or external work, the presence of the demon can be detected if the CI Eq. (2) does not hold. To challenge the limits of thermodynamics we assume that our demon is lazy. Even when the conditions are such that the demon should take action (i.e., shut the trap door) it may doze off and do nothing. Specifically, it will act a fraction $\chi$ of the times it should. When $\chi$ is sufficiently small $(\chi \leq \chi$ crit $)$, no anomalous heat will be observed. Thus, 
the action of the demon will not violate the CI, and the CI cannot be used to detect the demon.

Similarly to the discussion in Sec. III B, when the CI holds we cannot exclude the presence of feedback. In particular, there are two cases where this strategy can fail: (1) the feedback is very weak with respect to the flows generated by the thermodynamic protocol and (2) the elements (e.g., microbath) are initially correlated so the CI fails even without feedback. In this latter case, we can check for violation of the ICCI Eq. (25). Yet, in this case as well, the feedback may be too weak to violate the ICCI. Therefore, we focus here on the weak feedback scenario (small $\chi$ ).

The question we pose is, can thermodynamics be used to detect lazy demons that the CI cannot detect $\left(\chi<\chi_{\text {crit }}\right)$ ? Below, we present an example that demonstrates that the global passivity inequalities Eq. (30) can do the job.

In quantum mechanics, feedback can be described in the following way. The demon measures an observable with outcome $k$. For simplicity, we consider standard projective measurements (see Ref. [46] for feedback with weak measurement in thermodynamics). The measurement operation is described by a projection operator $\Pi_{k}^{2}=\Pi_{k}$. Depending on the result $k$, the experimentalist applies different unitaries $V_{k}$. The final density matrix with feedback is given by

$$
\rho_{f}^{\mathrm{tot}}=\sum_{k^{\prime}} V_{k^{\prime}} \Pi_{k^{\prime}}\left[\sum_{k} p_{k} U_{k} \rho_{0}^{\mathrm{tot}} U_{k}^{\dagger}\right] \Pi_{k^{\prime}} V_{k^{\prime}}^{\dagger},
$$

where the expression in the square bracket is $\rho_{f}^{\text {tot }}$ just before the feedback. While both Eqs. (6) and (39) are Kraus maps, Eq. (39) is not a mixture of unitaries due to the presence of the measurement projectors. Hence, the inequalities Eq. (30) are guaranteed to hold for Eq. (6) while for Eq. (39) they may be violated $\left(\Delta\left\langle\mathcal{B}^{\alpha}\right\rangle<0\right)$. This can be used to detect whether a feedback has been applied to the system.

Obviously, if for a specific $\alpha$ the inequality $\Delta\left\langle\mathcal{B}^{\alpha}\right\rangle \geq 0$ holds, the presence of a demon cannot be excluded, and other $\alpha$ values should be checked. The interesting question about the existence of a sufficient set of tests to detect any feedback is outside the scope of the present paper and will be explored elsewhere. Our more modest goal here is simply to demonstrate that in some cases the global passivity inequalities Eq. (30) are more sensitive compared to the CI, and can detect weak feedback that the CI cannot detect.

To illustrate how the global passivity inequalities Eq. (30) can assist in feedback detection, we study a simple setup of two microbaths composed of two spins each [Fig. 6(a)]. The cold (hot) microbath is initially prepared in temperature $T_{c}=1.5 \quad\left(T_{h}=2.5\right)$. The Hamiltonian of the setup before the interaction is
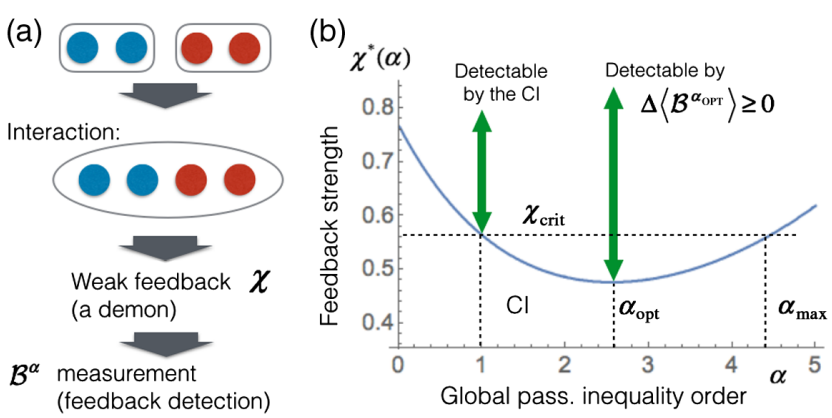

FIG. 6. (a) A schematic description of a process with a weak feedback ("lazy demon"). A hot and cold microbath are coupled, and evolve unitarily for a finite time. Then a feedback (see text) is applied to the setup with probability $\chi$ (feedback strength). (b) Lazy demon detection using global passivity inequalities Eq. (30). When the feedback strength exceeds $\chi_{\text {crit }}$, the heat changes its sign, and flows from the (initially) cold qubits to the hot qubits. In this case the action of the demon can be detected by a violation of CI. For $\chi \leq \chi_{\text {crit }}$, the CI cannot detect the demon. The blue curve depicts the value of $\chi^{*}(\alpha)$ for which $\Delta\left\langle B^{\alpha}\right\rangle=0$. The global passivity inequalities Eq. (30) can detect the demon for $\chi \geq \chi^{*}(\alpha)$ (right green arrow). Since for $1 \leq \alpha \leq \alpha_{\max }$, $\chi^{*}(\alpha) \leq \chi_{\text {crit }}$, we conclude that global passivity can be used to detect the demon that $\mathrm{CI}$ cannot. Interestingly, the best detection takes place at a noninteger value $\alpha_{\mathrm{opt}} \simeq 2.6$.

$H_{0}=\sum_{i=1}^{4} \sigma_{z}^{(i)}$. The initial state of the setup is $\rho_{0}^{\text {tot }}=\exp \left[-\beta_{c}\left(\sigma_{z}^{(1)}+\sigma_{z}^{(2)}\right)-\beta_{h}\left(\sigma_{z}^{(3)}+\sigma_{z}^{(3)}\right)\right] / Z$. At $t=0$ an "all to all" interaction is turned on: $H_{I}=\sum_{i>j} \sigma_{+}^{(i)} \sigma_{-}^{(j)}+$ $\sigma_{-}^{(i)} \sigma_{+}^{(j)}$. Such "all to all" coupling can be realized in ion traps or in superconducting qubits. The system evolves until $t=1$ under the Hamiltonian $H=H_{0}+H_{I}$. After the evolution, the demon is awake with probability $\chi$ and then it measures the setup. If the demon finds the setup to be in the state $\left|\uparrow_{c} \uparrow_{c} \downarrow_{h} \downarrow_{h}\right\rangle$, it changes it to $\left|\downarrow_{c} \downarrow_{c} \uparrow_{h} \uparrow_{h}\right\rangle$ (where $\uparrow_{\text {and }}$ $\downarrow$ stand for spin-up or spin-down states). In all other cases, the demon does nothing. This operation is energy conserving and transfers energy (heat) from the cold microbath to the hot microbath. Thus, no work is involved in applying this feedback. The process is shown in Fig. 6(a). Numerically, we apply the feedback and check if it is detectable. For $\chi=0$ (the demon never does anything) heat flows to the cold microbath, and the dynamics is consistent with the CI. Thus, for small values of $\chi$ this very subtle form of feedback may not be detectable by the CI.

Since our setup includes two microbaths and no system, the inequality Eq. (30) with $\alpha=1$ is identical to the CI Eq. (14): $\beta_{c} q_{q}+\beta_{h} q_{h} \geq 0$. We find that for $\chi$ values exceeding $\chi_{\text {crit }} \simeq 0.56$, heat flows from the cold microbath to the hot microbath and the demon is detectable by the CI Eq. (14). For other $\alpha$ values, we denote by $\chi^{*}(\alpha) \doteq$ $\min _{\chi}\left(\Delta\left\langle\mathcal{B}^{\alpha}\right\rangle<0\right)$ the smallest feedback strength that leads to violation of $\Delta\left\langle\mathcal{B}^{\alpha}\right\rangle \geq 0$. The blue curve in Fig. 6(b) shows $\chi^{*}(\alpha)$. Interestingly, this curves is not necessarily 
monotonically decreasing with $\alpha$. For this feedback operation, we find that among the $\alpha$ inequalities Eq. (30) there is an optimal value $\alpha_{\mathrm{opt}} \simeq 2.6$, where the largest detection range is observed (right green arrow). In this example, the best detection takes place for a noninteger value of $\alpha$. This gives a justification for studying also fractional values of $\alpha$.

Figure 6(b) shows that the global passivity inequalities Eq. (30) successfully detect "lazy demons" that the CI cannot detect. As in Sec. IV, these results demonstrate that different values of $\alpha$ contain different physical information.

\section{CONCLUSION}

The second law of thermodynamics is one of the pillars of theoretical physics with countless applications in engineering and science. It was originally formulated for macroscopic systems and reservoirs where one could assume weak coupling, extensivity, and lack of recurrences. Under some restrictions, the second law can also be applied to microscopic systems and microscopic environments. However, these restrictions exclude important microscopic setups. In other microscopic scenarios (e.g., dephasing), the second law is valid but provides trivial information that is of little use.

In this paper, we introduce the notion of global passivity. We show that under the standard thermodynamic assumptions global passivity (complemented by a passivitydivergence relation) recovers the Clausius inequality formulation of the second law. We then show that global passivity leads to a modified Clausius inequality that remains valid even when the system and environment are initially strongly correlated. This extension is important since for small systems the interaction between system and environment and the resulting initial correlations are, in general, non-negligible and the standard second law (in particular, the Clausius inequality form) cannot be used.

Our second main finding is a continuous family of global passivity inequalities that accompany the second law. We demonstrate how they can be used to detect heat leaks and feedback (demons) in cases where the second law fails to do so. In addition, the same inequalities are used to put upper and lower bounds on the buildup of systemenvironment covariance in a dephasing scenario. Such predictions are presently outside the scope of other modern thermodynamic frameworks (e.g., stochastic thermodynamics, and thermodynamic resource theory).

Beyond the two main findings of this paper, the global passivity formalism presents a set of tools that impose restrictions on observables in thermodynamic processes in quantum systems. As an example, in a different publication, we study the intimate relation between passivitydivergence relations and quantum coherence measures. Further research is needed in order to identify which inequalities are the most useful for a given setup.

The present predictions of global passivity are relevant to present-day experimental setups such as ion traps, superconducting circuits, optical lattices, and NMR.

\section{ACKNOWLEDGMENTS}

This work was supported by the U.S.-Israel Binational Science Foundation (Grant No. 2014405), by the Israel Science Foundation (Grant No. 1526/15), and by the Henri Gutwirth Fund for the Promotion of Research at the Technion. We thank Professor C. Jarzynski for pointing out the relation between the ICCI in the couple thermal state, and a recent classical result for a similar scenario [33].

\section{APPENDIX A: ALL OBSERVABLE ANALOG OF THE CLAUSIUS INEQUALITY}

Let us start with the standard initial condition of thermodynamic setups [assumptions (1) and (2) in Sec. II A]:

$$
\rho_{0}^{\mathrm{tot}}=\rho_{0}^{\mathrm{sys}} \otimes \rho^{-\sum \beta_{k} H_{k}} / Z,
$$

where $Z$ is a normalization factor, $H_{k}$ is the Hamiltonian of microbath $k$, and $\beta_{k}$ is its inverse temperature at time zero. Substituting Eq. (A1) in the global passivity inequality Eq. (17) gives

$$
\Delta\left\langle\mathcal{B}^{\text {sys }}\right\rangle+\sum_{k} \beta_{k} q_{k} \geq 0,
$$

where

$$
\mathcal{B}^{\text {sys }} \doteq-\ln \rho_{0}^{\text {sys }} .
$$

Equation (A2) is the observable-only analog of the CI. It contains only linear terms in the final density matrix. The $\mathrm{CI}$ and Eq. (A2) both reduce to $\sum_{k} \beta_{k} q_{k} \geq 0$ in two important cases: (1) when there is no mediating system (e.g., in absorption refrigerators with a tricycle interaction $[12,47]$ ) and (2) in (quasi)stationary periodic operation of a heat machine interacting with a large bath, where $\Delta\left\langle\mathcal{B}^{\text {sys }}\right\rangle$ is negligible with respect to the accumulated heat exchanges.

Despite the similarities, Eq. (A2) and the CI differ on a fundamental level. Equation (A2) contains only changes in expectation values of operators. Alternatively stated, it is linear in the final density matrix $\rho_{f}^{\text {tot }}$. It is nonlinear in $\rho_{0}^{\text {tot }}$, but this is due to the fact that the observable $\mathcal{B}$ is constructed from $\rho_{0}^{\text {tot }}$. In contrast, the term $\Delta S^{\text {sys }}$ in the $\mathrm{CI}$ does not have an expectation value structure and it is a nonlinear function of $\rho_{f}^{\text {tot }}$.

Even though the CI and Eq. (A2) involve different quantities, they can be quantitatively compared, as both provide a prediction on the quantity $\sum \beta_{k} q_{k}$. Using the identity Eq. (18) for the system density matrix, Eq. (A2) can be recast as

$$
\Delta S^{\mathrm{sys}}+\sum_{k} \beta_{k} q_{k} \geq-D\left(\rho_{f}^{\mathrm{sys}} \mid \rho_{0}^{\mathrm{sys}}\right) .
$$


Since the right-hand side of Eq. (A1) is negative, it becomes evident that the CI Eq. (2) is a tighter inequality compared to Eq. (A2). On the other hand, it is important to emphasize that Eq. (A2) takes less resources to evaluate in comparison to the CI. To evaluate the $\Delta S^{\text {sys }}$ term in the CI a full system tomography is needed. In contrast, in Eq. (A2) we measure one observable of the system $\hat{B}^{\text {sys }}$. Only the diagonal elements of the density matrix in the basis of $\hat{B}^{\text {sys }}$ are needed. Moreover, we do not need to know explicitly the diagonal elements of $\rho^{\text {sys }} ;\left\langle\mathcal{B}^{\text {sys }}\right\rangle$ can be directly measured without evaluating the probability distribution in the $\mathcal{B}^{\text {tot }}$ basis. Thus, in terms of utility for experiments, Eq. (A2) is easier to check compared to the CI. That said, the CI offers some insight on the relationship between information (entropy) and energy that is absent in Eq. (A2).

\section{APPENDIX B: OBSERVABLES INSPIRED BY PASSIVITY}

The ICCI Eq. (24) is given in terms of expectation values that are determined by the initial density matrix. When the initial density matrix is related to some observable, the ICCI expectation values will also be related to this observable. For example, in thermodynamics, the microbaths are determined by their initial temperature and their Hamiltonian, so $\mathcal{B}^{\text {bath }}$ or $\mathcal{B}^{\text {tot }}-\mathcal{B}^{\text {sys }}$ can be related to the Hamiltonian. In this appendix, we address the general case where there is no prior knowledge about the operator associated with the setup preparation. We limit our discussion to two initially correlated parties. The extension to more parties is trivial.

$\mathcal{B}^{\text {tot }}=-\ln \rho_{0}^{\text {tot }}$ is a positive Hermitian operator, and it can be decomposed in the following way:

$$
\begin{aligned}
\mathcal{B}^{\mathrm{tot}}= & {\left[\sum_{i=1}^{L_{A}} r_{A, i} Z_{i} \otimes \frac{I_{N_{B} \times N_{B}}}{N_{B}}+\sum_{j=1}^{L_{A}} r_{B, i} \frac{I_{N_{A} \times N_{A}}}{N_{A}}\right.} \\
& \left.\otimes Z_{j}+\sum_{i=1}^{L_{A}-1} \sum_{j=1}^{L_{B}-1} t_{i j} Z_{i} \otimes Z_{j}\right]
\end{aligned}
$$

where $L_{A(B)}=N_{A(B)}^{2}-N_{A(B)}$, and $\left\{Z_{i(j)}\right\}_{1}^{L_{A(B)}-1}$ are traceless orthonormal basis operators for $N_{A(B)} \times N_{A(B)}$ Hermitian traceless matrices. $Z_{L_{A(B)}}=I_{N_{A(B)} \times N_{A(B)}}$ are the identity operators in each party. Finally, $r_{A}$ and $r_{B}$ determine the reduced density matrices:

$$
\operatorname{tr}_{B} \mathcal{B}^{\text {tot }}=\left[\sum r_{A, i} Z_{i}\right]=\mathcal{B}_{A}
$$

Decomposition (B1) suggests that $\mathcal{B}^{\text {tot }}=\mathcal{B}_{A}+\mathcal{B}_{B}+$ $\mathcal{B}_{\text {int }}$, where $\mathcal{B}_{\text {int }}$ has an interaction Hamiltonian form, $\mathcal{B}_{\text {int }}=\sum_{i j} t_{i j} Z_{i} \otimes Z_{j}$. Now let $A$ be the system and $B$ be the environment. We find that ICCI has the form:

$$
\Delta S^{\text {sys }}+\Delta\left\langle\mathcal{B}^{\text {bath }}\right\rangle+\Delta\left\langle\mathcal{B}^{\text {int }}\right\rangle+\Delta\left\langle\mathcal{B}^{\text {sys }}-\mathcal{B}_{\text {eff }}^{\text {sys }}\right\rangle \geq 0,
$$

where

$$
\begin{aligned}
\mathcal{B}^{\text {bath }} & =\operatorname{tr}_{\text {sys }}\left(-\ln \rho^{\text {tot }}\right), \\
\mathcal{B}^{\text {sys }} & =\operatorname{tr}_{\text {env }}\left(-\ln \rho^{\text {tot }}\right), \\
\mathcal{B}_{\text {eff }}^{\text {sys }} & =-\ln \left[\operatorname{tr}_{\text {env }}\left(\rho^{\text {tot }}\right)\right] .
\end{aligned}
$$

Thus, the dressing term originates from the noncommutativity of the partial trace operation and the $-\ln$ operation. The two commute when $\rho_{0}^{\text {tot }}$ is in a system-environment product state.

\section{APPENDIX C: ICCI FOR TWO SIMPLE INTERACTIONS IN A COUPLED THERMAL STATE}

In this appendix, we evaluate the magnitude of the system dressing term in the ICCI with an initially coupled thermal state (see Sec. II E), $\beta\left(H_{s}-H_{s}^{\text {eff }}\right)$, for two interesting cases. First, we make the simplifying assumption that $H_{I, 0}$ conserves the sum of bare energy of the system and the microbath (denoted by ' $b$ ' for brevity):

$$
\left[H_{I, 0}, H_{s}+H_{\mu b}\right]=0 \text {. }
$$

Using this condition, we find

$$
\begin{aligned}
\operatorname{tr}_{h} e^{-\beta\left(H_{s}+H_{I, 0}+H_{b}\right)} & =e^{-\beta H_{s}} \operatorname{tr}_{h}\left[e^{-\beta H_{b}} e^{-\beta H_{I, 0}}\right] \\
& =e^{-\beta H_{s}} \sum_{E_{b}} e^{-\beta E_{b}}\left\langle E_{b}\left|e^{-\beta H_{I, 0}}\right| E_{b}\right\rangle,
\end{aligned}
$$

where in the last stage we have used the microbath energy eigenstates to do the partial trace.

We consider two typical forms of $H_{I, 0}$ : (1) $\epsilon \mathcal{H}_{s} \otimes \mathcal{H}_{b}$, where $\left[\mathcal{H}_{b}, H_{b}\right]=\left[\mathcal{H}_{s}, H_{s}\right]=0$, which often arises in dephasing environments, e.g., $\sigma_{z} \otimes \sigma_{z}$, and (2) a swaplike interaction between the system and the microbath $\epsilon\left(a_{b}^{\dagger}\right) \otimes a_{s}+$ H.c., where $a$ is some transition operator of the form $|m\rangle\langle n|$.

For case (1), simple dephasing, we find

$$
\begin{aligned}
\operatorname{tr}_{h} e^{-\beta\left(H_{s}+H_{h}+\epsilon \mathcal{H}_{s} \otimes \mathcal{H}_{b}\right)} & =e^{-\beta H_{s}} \sum_{n=0} \frac{\left(-\beta \epsilon \mathcal{H}_{s}\right)^{n} \otimes \operatorname{tr}\left[e^{-\beta H_{b}} \mathcal{H}_{b}^{n}\right]}{n !} \\
& =e^{-\beta H_{s}} \sum_{n=0} \frac{\left(-\beta \epsilon \mathcal{H}_{s}\right)^{n}}{n !}\left\langle\mathcal{H}_{b}^{n}\right\rangle_{0} Z_{b 0}
\end{aligned}
$$

where $Z_{b 0}=\operatorname{tr}\left[e^{-\beta H_{b}}\right]$ and $\left\langle\mathcal{H}_{b}^{n}\right\rangle_{0}=\operatorname{tr}\left\{\left[\left(e^{-\beta H_{b}}\right) /\left(Z_{b 0}\right) \mathcal{H}_{b}^{n}\right]\right\}$. We define the function $f(x)=\sum_{n=0}\left[\left(Z_{b 0}\left\langle\mathcal{H}_{b}^{n}\right\rangle_{0}\right) / n !\right] x^{n}$, so from Eqs. (28) and (C3) we get 


$$
\begin{aligned}
\beta \Delta\left(H_{\mathrm{eff}}^{\mathrm{sys}}-H_{s}\right) & =\ln f\left(-\beta \epsilon \mathcal{H}_{s}\right) \\
& =(\beta \epsilon) \mathcal{H}_{s}\left\langle\mathcal{H}_{b}\right\rangle_{0} Z_{b 0}+O\left[(\beta \epsilon)^{2}\right] .
\end{aligned}
$$

Since $\mathcal{H}_{s}$ commute with $H_{s}$, we conclude that $\ln f\left(-\beta \epsilon \mathcal{H}_{s}\right)$ is a "dressing effect" of the microbath on the system (this term has the same eigenstates as $H_{s}$ ). In the ICCI Eq. (24), the system appears primarily in $\Delta S^{\mathrm{sys}}$, as in the CI. However, in the ICCI the dephasing dressing effect contributes a new expectation value term (in contrast to information term) of the system Eq. (C4).

For case (2), the creation-annihilation swaplike interaction, we find

$$
\begin{aligned}
\operatorname{tr}_{b} e^{-\beta\left(H_{s}+H_{I, 0}+H_{b}\right)} & =e^{-\beta H_{s}} \sum_{E_{b}} e^{-\beta E_{b}}\left\langle E_{b}\left|e^{-\beta H_{I, 0}}\right| E_{b}\right\rangle \\
& =e^{-\beta H_{s}} \sum_{E_{b}} e^{-\beta E_{b}}\left\langle E_{b}\left|\cosh \left(\beta H_{I, 0}\right)\right| E_{b}\right\rangle .
\end{aligned}
$$

The cosh appears since all the odd powers of $e^{-\beta H_{I, 0}}$ are eliminated by the partial trace. From this we conclude that the contribution of the swap term is $O\left[(\beta \epsilon)^{2}\right]$. Therefore, if there is a weak swap term at time zero, it will manifest in the first order in the $\beta \Delta\left\langle H_{I, 0}\right\rangle$ term of the ICCI, and only in second order in the system dressing term of the ICCI.

\section{APPENDIX D: $\mathcal{B}^{\alpha}$ HIERARCHY RELATIONS}

In this appendix, we point out that there is a hierarchy relation between inequalities Eq. (30) with different values of $\alpha$. The derivation is based on the fact that for any $q>p>0$, the function $h(x)=\left(x^{p} / p\right)-\left(x^{q} / q\right)$ is monotonically increasing for $x \in[0,1]$. To apply this to the operator $\mathcal{B}=-\ln \rho_{0}^{\text {tot }}$, we define the operator $\tilde{\mathcal{B}}=\mathcal{B} /\|\mathcal{B}\|_{\text {op }}$, where $\|\mathcal{B}\|_{\text {op }}$ is the operator norm, which is equal to the largest eigenvalue of $\mathcal{B}(\mathcal{B}$ is Hermitian and bounded). Based on this construction $h(\tilde{\mathcal{B}})$ is passive with respect to $\rho_{0}^{\text {tot }}$ since we ensured that the spectrum of $\tilde{\mathcal{B}}$ is in $[0,1]$, where $h(x)$ is an increasing function. Thus, from passivity we get that $\Delta h(\tilde{\mathcal{B}}) \geq 0$, which means that

$$
\frac{\Delta\left\langle\mathcal{B}^{p}\right\rangle}{p\|\mathcal{B}\|_{\mathrm{op}}^{p}} \geq \frac{\Delta\left\langle\mathcal{B}^{q}\right\rangle}{q\|\mathcal{B}\|_{\mathrm{op}}^{q}}, \quad \forall p>q>0 .
$$

In particular, for integer $p$ and $q$ we get

$$
\frac{\Delta\langle\mathcal{B}\rangle}{\|\mathcal{B}\|_{\text {op }}} \geq \frac{1}{2} \frac{\Delta\left\langle\mathcal{B}^{2}\right\rangle}{\|\mathcal{B}\|_{\text {op }}^{2}} \geq \frac{1}{3} \frac{\Delta\left\langle\mathcal{B}^{3}\right\rangle}{\|\mathcal{B}\|_{\mathrm{op}}^{3}} \geq \cdots \geq \frac{1}{n} \frac{\Delta\left\langle\mathcal{B}^{n}\right\rangle}{\|\mathcal{B}\|_{\text {op }}^{n}} \geq 0 .
$$

These relations can be useful for bounded setups (e.g., collection of spins) as long as the operator $\mathcal{B}$ is bounded and not too large (e.g., when the setup includes a small number of spins). For large $\|\mathcal{B}\|_{\text {op }}$ the hierarchy relations Eq. (D1) reduce to Eq. (30).
[1] D. Jennings and T. Rudolph, Entanglement and the Thermodynamic Arrow of Time, Phys. Rev. E 81, 061130 (2010).

[2] K. Micadei, J. P. S. Peterson, A. M. Souza, R. S. Sarthour, I. S. Oliveira, G. T. Landi, T. B. Batalhão, R. M. Serra, and E. Lutz, Reversing the Thermodynamic Arrow of Time Using Quantum Correlations, arXiv:1711.03323.

[3] U. Seifert, Stochastic Thermodynamics, Fluctuation Theorems and Molecular Machines, Rep. Prog. Phys. 75, 126001 (2012).

[4] K. Sekimoto, Stochastic Energetics (Springer, New York, 2010), Vol. 799.

[5] C. Jarzynski, Equalities and Inequalities: Irreversibility and the Second Law of Thermodynamics at the Nanoscale, Annu. Rev. Condens. Matter Phys. 2, 329 (2011).

[6] R. J. Harris and G. M. Schütz, Fluctuation Theorems for Stochastic Dynamics, J. Stat. Mech. (2007) P07020.

[7] G. Gour, M. P. Müller, V. Narasimhachar, R. W. Spekkens, and N. Y. Halpern, The Resource Theory of Informational Nonequilibrium in Thermodynamics, Phys. Rep. 583, 1 (2015).

[8] F. Brandão, M. Horodecki, N. Ng, J. Oppenheim, and S. Wehner, The Second Laws of Quantum Thermodynamics, Proc. Natl. Acad. Sci. U.S.A. 112, 3275 (2015).

[9] M. Horodecki and J. Oppenheim, Fundamental Limitations for Quantum and Nanoscale Thermodynamics, Nat. Commun. 4, 2059 (2013).

[10] M. Lostaglio, D. Jennings, and T. Rudolph, Description of Quantum Coherence in Thermodynamic Processes Requires Constraints beyond Free Energy, Nat. Commun. 6, 6383 (2015).

[11] J. Roßnagel, S. T. Dawkins, K. N. Tolazzi, O. Abah, E. Lutz, F. Schmidt-Kaler, and K. Singer, A Single-Atom Heat Engine, Science 352, 325 (2016).

[12] G. Maslennikov, S. Ding, R. Hablutzel, J. Gan, A. Roulet, S. Nimmrichter, J. Dai, V. Scarani, and D. Matsukevich, Quantum Absorption Refrigerator with Trapped Ions, arXiv: 1702.08672 .

[13] P. O. Boykin, T. Mor, V. Roychowdhury, F. Vatan, and R. Vrijen, Algorithmic Cooling and Scalable NMR Quantum Computers, Proc. Natl. Acad. Sci. U.S.A. 99, 3388 (2002).

[14] W. S. Bakr, P. M. Preiss, M. E. Tai, R. Ma, J. Simon, and M. Greiner, Orbital Excitation Blockade and Algorithmic Cooling in Quantum Gases, Nature (London) 480, 500 (2011).

[15] J. Klatzow, C. Weinzetl, P. M. Ledingham, J. N. Becker, D. J. Saunders, J. Nunn, I. A. Walmsley, R. Uzdin, and E. Poem, Experimental Demonstration of Quantum Effects in the Operation of Microscopic Heat Engines, arXiv:1710.08716.

[16] W. Pusz and S. L. Wornwicz, Passive States and KMS States for General Quantum Systems, Commun. Math. Phys. 58, 273 (1978).

[17] A Lenard, Thermodynamical Proof of the Gibbs Formula for Elementary Quantum Systems, J. Stat. Phys. 19, 575 (1978).

[18] A. E. Allahverdyan, R. Balian, and Th. M. Nieuwenhuizen, Maximal Work Extraction from Finite Quantum Systems, Eurphys. Lett. 67, 565 (2004).

[19] M. Perarnau-Llobet, K. V. Hovhannisyan, M. Huber, P. Skrzypczyk, N. Brunner, and A. Acín, Extractable Work from Correlations, Phys. Rev. X 5, 041011 (2015). 
[20] W. Niedenzu, V. Mukherjee, A. Ghosh, A. G. Kofman, and G. Kurizki, Quantum Engine Efficiency Bound Beyond the Second Law of Thermodynamics, Nat. Commun. 9, 165 (2018).

[21] T. Sagawa, Second Law-like Inequalities with Quantum Relative Entropy: An Introduction, Lect. Quantum Comput. Thermodyn. Stat. Phys. 8, 127 (2012).

[22] A. Peres, Quantum Theory: Concepts and Methods (Springer Science \& Business Media, Netherlands, 2006), Vol. 57.

[23] M. Esposito, K. Lindenberg, and C. Van den Broeck, Entropy Production as Correlation Between System and Reservoir, New J. Phys. 12, 013013 (2010).

[24] M. Esposito and C. Van den Broeck, Second Law and Landauer Principle Far from Equilibrium, Europhys. Lett. 95, 40004 (2011).

[25] P. Kammerlander and J. Anders, Coherence and Measurement in Quantum Thermodynamics, Sci. Rep. 6, 22174 (2016).

[26] R. Uzdin, Additional Energy-Information Relations in Thermodynamics of Small Systems, Phys. Rev. E 96, 032128 (2017).

[27] M. Perarnau-Llobet, K. V. Hovhannisyan, M. Huber, P. Skrzypczyk, J. Tura, and A. Acín, Most Energetic Passive States, Phys. Rev. E 92, 042147 (2015).

[28] In small quantum systems the coarse graining is obtained by the partial trace operation that leads to a reduced (local) description. This reduced description ignores the presence of the correlation between the elements, so the sum of entropies is not fixed in time. However, the von Neumann entropy of the whole setup $S\left(\rho^{\text {tot }}\right)$ contains the correlations, and since it is unitarily invariant, it holds that $S\left(\rho_{f}^{\mathrm{tot}}\right)=S\left(U \rho_{0}^{\mathrm{tot}} U^{\dagger}\right)=S\left(\rho_{0}^{\mathrm{tot}}\right)$.

[29] A. W. Marshall, I. Olkin, and B. C. Arnold, Inequalities: Theory of Majorization and Its Applications (Springer, New York, 1979), Vol. 143.

[30] M. A. Nielsen and I. L. Chuang, Quantum Computation and Quantum Information (Cambridge University Press, Cambridge, England, 2004).

[31] C. Jarzynski, Microscopic Analysis of Clausius-Duhem Processes, J. Stat. Phys. 96, 415 (1999).

[32] J. G. Kirkwood, Statistical Mechanics of Fluid Mixtures, J. Chem. Phys. 3, 300 (1935).

[33] C. Jarzynski, Stochastic and Macroscopic Thermodynamics of Strongly Coupled Systems, Phys. Rev. X 7, 011008 (2017).
[34] U. Seifert, First, and Second Law of Thermodynamics at Strong Coupling, Phys. Rev. Lett. 116, 020601 (2016).

[35] H. J. D. Miller and J. Anders, Entropy Production and Time Asymmetry in the Presence of Strong Interactions, Phys. Rev. E 95, 062123 (2017).

[36] S. Still, D. A. Sivak, A. J. Bell, and G. E. Crooks, Thermodynamics of Prediction, Phys. Rev. Lett. 109, 120604 (2012).

[37] M. N. Bera, A. Riera, M. Lewenstein, and A. Winter, Generalized Laws of Thermodynamics in the Presence of Correlations, Nat. Commun. 8, 2180 (2017).

[38] D. Reeb and M. M. Wolf, An Improved Landauer Principle with Finite-Size Corrections, New J. Phys. 16, 103011 (2014).

[39] S. Jevtic, T. Rudolph, D. Jennings, Y. Hirono, S. Nakayama, and M. Murao, Exchange Fluctuation Theorem for Correlated Quantum Systems, Phys. Rev. E 92, 042113 (2015).

[40] H. S. Leff and A. F. Rex, Maxwell's Demon: Entropy, Information, Computing (Princeton University Press, Princeton, NJ, 2014).

[41] J. M. R. Parrondo, J. M. Horowitz, and T. Sagawa, Thermodynamics of Information, Nat. Phys. 11, 131 (2015).

[42] S. Toyabe, T. Sagawa, M. Ueda, E. Muneyuki, and M. Sano, Experimental Demonstration of Information-to-Energy Conversion and Validation of the Generalized Jarzynski Equality, Nat. Phys. 6, 988 (2010).

[43] M. D. Vidrighin, O. Dahlsten, M. Barbieri, M. S. Kim, V. Vedral, and I. A. Walmsley, Photonic Maxwell's Demon, Phys. Rev. Lett. 116, 050401 (2016).

[44] J. V. Koski, V. F. Maisi, J. P. Pekola, and D. V. Averin, Experimental Realization of a Szilard Engine with a Single Electron, Proc. Natl. Acad. Sci. U.S.A. 111, 13786 (2014).

[45] A. Bérut, A. Arakelyan, A. Petrosyan, S. Ciliberto, R. Dillenschneider, and E. Lutz, Experimental Verification of Landauer's Principle Linking Information and Thermodynamics, Nature (London) 483, 187 (2012).

[46] A. Levy, L. Diósi, and R. Kosloff, Quantum Flywheel, Phys. Rev. A 93, 052119 (2016).

[47] P. P. Hofer, M. Perarnau-Llobet, J. B. Brask, R. Silva, M. Huber, and N. Brunner, Autonomous Quantum Refrigerator in a Circuit QED Architecture Based on a Josephson Junction, Phys. Rev. B 94, 235420 (2016).

Correction: The affiliation indicator for the second author was misarranged during the production cycle and has been corrected. 